\title{
Chromosomal architecture changes upon cell differentiation
}

\author{
Maxim Imakaev ${ }^{1 *}$, Geoffrey Fudenberg ${ }^{2}$, Leonid Mirny ${ }^{1,2,3}$ \\ From Epigenetics \& Chromatin: Interactions and processes \\ Boston, MA, USA. 11-13 March 2013
}

\section{Background}

The recently developed Hi-C method provides a comprehensive whole-genome picture of physical contacts between distal loci. Analysis of these data has begun to reveal determinants of 3D genomic organization. However, the similarities and differences in chromosomal organization between cell-types remain unexplored.

\section{Materials and methods}

To analyze chromosomal architecture between cell types, it is crucial to have a consistent way of analyzing $\mathrm{Hi}-\mathrm{C}$ data and removal of experimental biases. To this end we developed a comprehensive method of Iterative Correction and Eigenvector decomposition (ICE) ${ }^{2}$. ICE maps Hi-C reads to the genome, filters mapped reads and obtains a $\mathrm{Hi}-\mathrm{C}$ map of relative contact probabilities free of experimental biases. It then decomposes the maps into a set of genomic tracks characterizing high-order chromatin organization.

\section{Results}

Using ICE, we analyze Hi-C data ${ }^{3}$ from human embryonic stem cells, and IMR90 lung fibroblast cells. We focus our analysis on the compartment profile, which has been shown to partition the genome into transcribed gene-rich regions, enriched in active chromatin marks ("active" regions), and "inactive" gene-poor regions. First, we show that ES cells have a gradual transition between "active" and "inactive" chromatin interaction preferences, as demonstrated by a broad unimodal distribution of values of the compartment profile. In contrast, differentiated IMR90 cells show one inactive chromatin state and a range of states at the active end. Second, we find that chromatin interactions in embryonic cells are best described by GC

\footnotetext{
'Department of Physics, Massachusetts Institute of Technology, Cambridge, MA, USA

Full list of author information is available at the end of the article
}

content of a genomic region. Conversely, for the differentiated cell line IMR90, transcription data (CAGE) is a much better predictor of chromatin interaction preferences than sequence-derived features. Lastly, we analyze changes in chromatin interactions upon differentiation, and find that regions which belonged to an active compartment in ES cells often switch to inactive compartment in IMR90, while the opposite rarely happens.

\section{Conclusions}

Taken together, our results show that genome-wide chromatin interactions change upon differentiation of ES cells into IMR90, and suggest that sequence-dependent chromatin interactions in embryonic stem cells get overridden in a cell-type-specific manner. We show that upon differentiation regions change from an active to an inactive compartment, suggesting that change in chromatin interactions reflects cell-type-specific silencing of genomic regions.

\section{Author details}

${ }^{1}$ Department of Physics, Massachusetts Institute of Technology, Cambridge, MA, USA. ${ }^{2}$ Harvard University, Program! in Biophysics, Boston, Massachusetts, USA. ${ }^{3}$ Institute for Medical Engineering and Science, MIT, USA.

Published: 8 April 2013

\section{References}

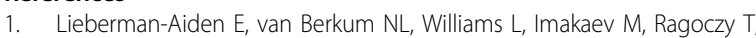
Telling A, Dekker J, et al: Comprehensive mapping of long-range interactions reveals folding principles of the human genome. Science 2009, 326(5950):289-293.

2. Imakaev M, Fudenberg G, McCord RP, Naumova N, Goloborodko A, Lajoie BR, Mirny LA, et al: Iterative correction of $\mathrm{Hi}-\mathrm{C}$ data reveals hallmarks of chromosome organization. Nature Methods 2012, 9(10):999-1003.

3. Dixon JR, Selvaraj S, Yue F, Kim A, Li Y, Shen Y, Ren B, et al: Topological domains in mammalian genomes identified by analysis of chromatin interactions. Nature 2012, 485(7398):376-338.

doi:10.1186/1756-8935-6-S1-P130

Cite this article as: Imakaev et al:: Chromosomal architecture changes upon cell differentiation. Epigentics \& Chromatin 2013 6(Suppl 1):P130. 